\title{
Why are Diverticula of the Rectum Uncommon? Angiomorphological Hypothesis
}

\author{
Bernardes A*, Melo C, Moura D and Pinheiro S \\ Associate Professor, University of Coimbra, Portugal \\ *Corresponding author: Antonio Bernardes, Associate Professor, University of Coimbra, Rua Antonio Jardim, Urbanizacao Quinta do Cedro, lote 1, \\ 3000-036 Coimbra-Portugal, Tel: 00351919695799, Email: antoniojbernardes@gmail.com
}

Submission: 1 望 December 18, 2017; Published: 些 December 21, 2017

\begin{abstract}
Introduction/Objectives: The cause of rectal diverticula is still unknown and its incidence is very low when compared to the incidence of diverticular disease in the colon. The aim of this work is to propose an angiomorphological etiology to complement the theories already described to explain the low incidence of diverticular disease of the rectum and the rare occurrence of diverticula in the mesocolicside of the sigmoid colon: a hypothesis based on the location and course of the arteries through the colon wall and in the diameter of the lumen of the colon.

Material and methods: Eighty specimens retrieved from donated human cadavers were used. They were all injected via the inferior mesenteric artery with colloidal barium sulfate with red colored gelatin. Ten cases were also injected via the two internal iliac arteries. All of them were then preserved using the Spalteholz diaphanization technique. In four cases was also used a micro-angiography technique.

Results: The vasa recta longa (VRL) course through only one muscle layer, the inner circular muscle layer, during a short tract, almost perpendicular to the greater axis of the colon, and then end in the submucosa. Most of the vasa recta brevia (VRB) cross the two muscle layers at the level of the mesocolic taeniae. The terminal branches of the superior rectal artery cross the two muscular layers of the rectum, from the external towards the inner layer, oriented longitudinally and obliquely during a long tract through the rectal wall until they reach the submucosa.

Conclusion: The areas where the VRL course only through the circular muscle layer are weak points of the colon wall where the diverticula can frequently occur. We think that the lower incidence of diverticula in the mesocolic border of the colon is due to the fact that the VRB have a smaller caliber than the VRL, they cross more often the two muscle layers (at the mesocolictænia level) and, in the cases where they cross only the muscle circular layer, they have an oblique path for a longer tract. Diverticula are rare in the rectum not only for the various reasons already described but also because the arteries that supply the rectum cross the wall of the organ in an oblique and longitudinal way, for a long tract.
\end{abstract}

\section{Introduction}

The incidence of diverticula in the rectum is very low, ranging from 0.07 to $0.08 \%$ [1], compared to rates of 20 to $40 \%$ observed in the colon, despite the intra luminal pressures observed in the rectal lumen. The diverticula of the colon are called pseudo-diverticula. They are small (5 to $10 \mathrm{~mm}$ ), appear in multiple numbers (may be hundreds) and occur preferentially in the areas of penetration of vasa recta through the wall of the organ [2]. The natural history and patho-physiology of diverticular disease is complex and continues to be not fully understood nowadays, being considered multi factorial [2]. Most of the rectal diverticula are true diverticula, usually solitary ( 1 to 3 per case) and large ( $2 \mathrm{~cm}$ in diameter) [3]. The etiology of rectal diverticula is still unknown [4]. They occur in a higher frequency in the lateral wall of the rectum, where the longitudinal muscle layer is thinner. They are observed in 2 to $2.4 \%$ of the cases of colon diverticulosis [5] and occur sometimes associated with scleroderma. In order to explain the low incidence of rectal diverticula, several hypotheses have been proposed: the protection offered by the perirrectal muscle-fibrous structures, the presence of a continuous circumferential muscle layer (the circular and longitudinal muscle layers) and the lesser peristaltic activity in the rectum $[2,4]$.

\section{Objectives}

This work proposes an angiomorphologicaletiology to complement the theories already described to explain the low incidence of diverticular disease of the rectum and the rare occurrence of diverticula in the mesocolicside of the sigmoid colon: a hypothesis based on the location and course of the arteries through the colon wall and in the diameter of the lumen of the colon.

\section{Material and Methods}

Eighty specimens collected from human cadavers were used. Two groups were formed: 60 cases ( $1^{\text {st }}$ group) included the sigmoid colon, rectum and anal canal and the remaining 20 cases ( $2{ }^{\text {nd }}$ group) included only the rectossigmoid transitions and the rectal and anal canal transitions. The specimens were all injected via the inferior mesenteric artery with colloidal barium sulfate with red colored 
gelatin. Ten cases of the $2^{\text {nd }}$ group were also injected via the two internal iliac arteries with different dyes: red dye on the right and green dye on the left. All organs were then preserved using the Spalteholz diaphanization (Figure 1 \& 2) technique (clearing method) [6] and in four cases it was also used a microangiography technique [7].

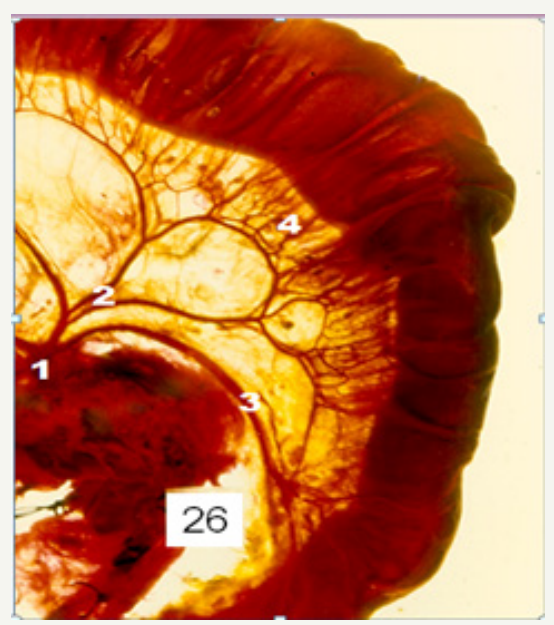

Figure 1: Diaphanization technique of the human colon sigmoid and rectum: 1-Inferior mesenteric artery; 2-Sigmoid arteries; 3-Superior rectal artery; 4-Vasa reta.

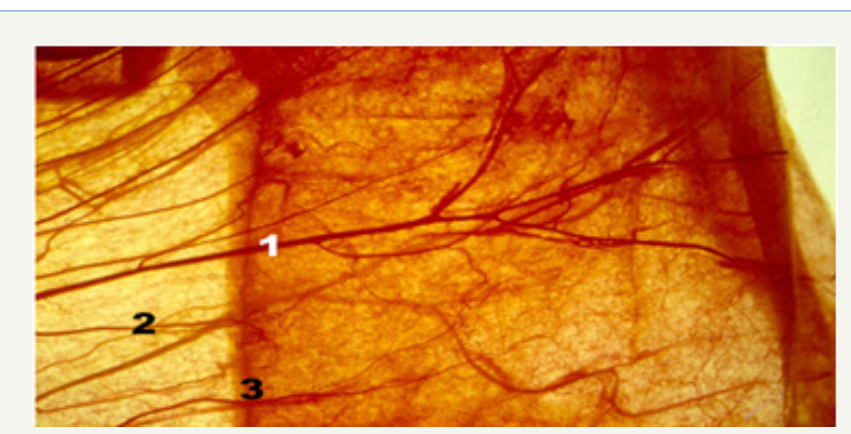

Figure 2: Diaphanization technique of the human colon sigmoid: 1-Vasa recta longa; 2-Vasa recta brevia; 3-Mesocolic border.

\section{Results}

In the sigmoid colon, the marginal artery that provides arterial supply, gives rise to two types of vasa recta: long and brevia. The vasa recta longa (VRL), after their origin in the marginal artery in a straight angle, travel almost describing a rectilinear tract in the thickness of the mesocolon and reach the wall of the colon. They then course though the subserouslayer perpendicular to the largest axis of the colon between the mesocolic border and the taeniae coli libera, towards the free border of the colon. When the VRL reach the inner edge of the taeniae coli libera changes direction, leaves the subserosa, crosses only one muscle layer during a short tract, the inner circular muscle layer, almost perpendicular to the greater axis of the colon, and ends in the submucosa. However, $40.9 \%$ of the VRL end during their tract through the mesocolon. Therefore, in these cases, there is a terminal branch of the VRL that crosses the wall of the colon. The VRL have an average caliber of
$620.2 \pm 42.34 \mu \mathrm{m}$ (limits: 300 and $800 \mu \mathrm{m}$ ) in their origin, but when they reach the anti-mesocolictaeniae they have an average caliber of $367.2 \pm 89.99 \mu \mathrm{m}$. During their tract the VRL give origin to two types of collateral branches: mesocolonic (those who are originated in the mesocolon) and colic or parietal (when they are originated in the wall of the colon). The parietal branches can be wet called subserous, epiploic, intestinal, submucous and muscle recurrent [7].

The vasa recta brevia (VRB) exist in greater number than the VRL. They arise from the marginal artery or, more frequently, from the VRL during their tract in the thickness of the mesocolon, where they travel until reaching the mesocolic border of the colon. Most VRB (74.9\%) cross all visceral layers including the taeniae coli mesocolicain a short tract and end in the submucosa. A small percentage of VRB $(25.1 \%)$ crosses only the circular muscle layer, next to the taeniae coli mesocolica, and also ends in the submucosa. The VRB have an average caliber of $190.3 \pm 29.83 \mu$ m (limits: 187.3 and $193.3 \mu \mathrm{m}$ ). During their tract the VRB gives rise to two types of collateral branches: mesocolic and colonic or parietal (Figure 3 \& 4).

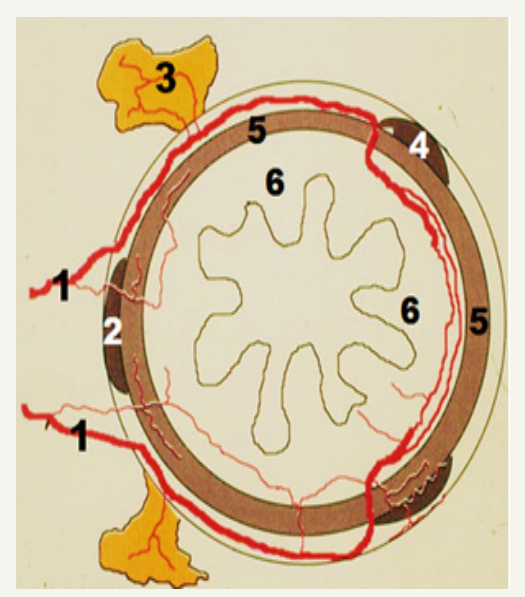

Figure 3: Colon vascularization scheme. Traject of the vasa recta longa in the colon wall: 1-Vasa recta longa; 2-Taeniae coli mesocólica; 3-Epiploic appendice; 4-Free taeniae coli; 5-Circular muscle layer; 6-Submucosa.

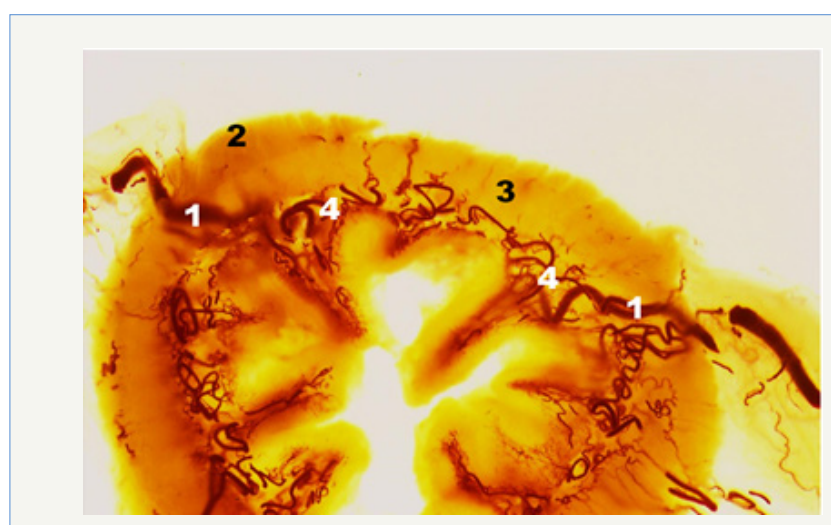

Figure 4: Diaphanization technique of the human colon sigmoid: 1-Vasa recta longa; 2-Taeniae coli mesocólica; 3-Circular muscle layer; 4-Submucosa. 
The parietal branches may be named subserosal, muscular and submucosal. The collateral and terminal branches of the VRL and VRB establish longitudinal and transverse anastomoses in the colonic wall, forming four rich and homogeneous plexuses: subserous, muscular, submucous and mucous. In the rectum the superior rectal artery, terminal branch of the inferior mesenteric artery, walks downwards and forwards inside the mesorrectum and reaches the posterior wall of the rectum. In most cases the superior rectal artery ends in the thickness of the mesorrectum near the posterior wall of the rectum by bifurcation $(81.3 \%)$ or trifurcation. Each of these terminal branches has a downward oblique path along the posterior and lateral surfaces of the rectum. These terminal branches of the superior rectal artery cross the two muscle layers of the rectum from the external to the internal layer and are oriented downwards, longitudinally and obliquely during a long tract. These terminal branches of secondary division of the superior rectal artery have an average caliber of $0.7 \pm 0.24 \mathrm{~mm}$ (limits: 0.67 and $1.1 \mathrm{~mm}$ ). They reach the submucosal layer at about $8 \mathrm{~cm}$ from the anal margin. Then they divide again into branches that reach the submucosa of the anal canal. The rectum also receives accessory vascularization from the middle rectal arteries, which are inconstant branches of the internal iliac. In fact they are generally bilateral but may be unilateral (Figure 5).

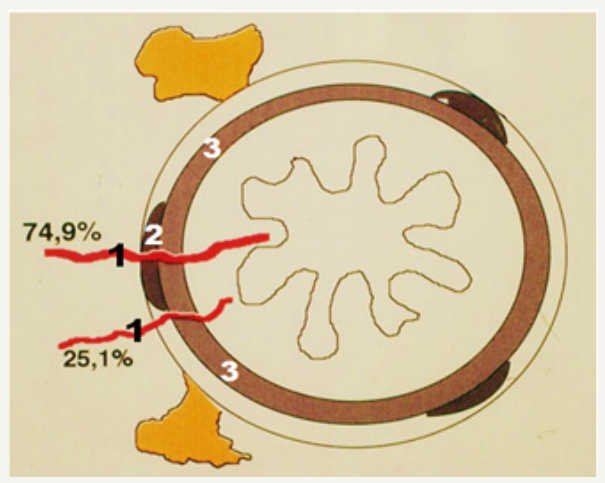

Figure 5: Colon vascularization scheme. Traject of the vasa recta breviain the colon wall: 1-Vasa recta brevia; 2Taeniae coli mesocólica; 3-Circular muscle layer.

\section{Discussion}

The classic description of the colon diverticulum is a hernia composed of mucosa, submucosa and serosa, passing through the muscular circular layer [2]. Diverticula arise preferentially in the sigmoid colon, where the VRLcrosses only the circular musclelayer perpendicular to the major axis of the colon towards the submucosa, near the free border of the colon. These sites where the VRL cross only one muscle layer are points of smaller resistance where mucosal hernias may occur (that is: diverticula), especially when there is a chronically increased endoluminal pressure. At the level of the mesocolic border there are also vasa recta, the VRB, but there is a lower incidence of diverticula. This fact can be explained by three reasons:

1. The muscular structure of the wall of the colon in that region

\section{The caliber of the vessels}

3. The tract of the vessels.

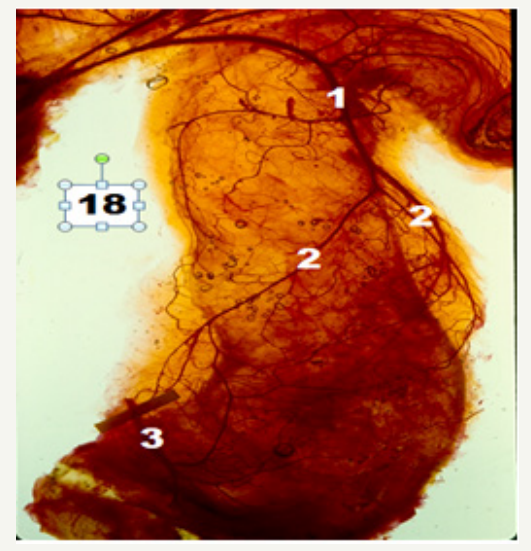

Figure 6: Diaphanization technique of the human rectum: 1-Superior rectal artery; 2-Terminal branches of the superior rectal artery; 3-Middle rectal artery.

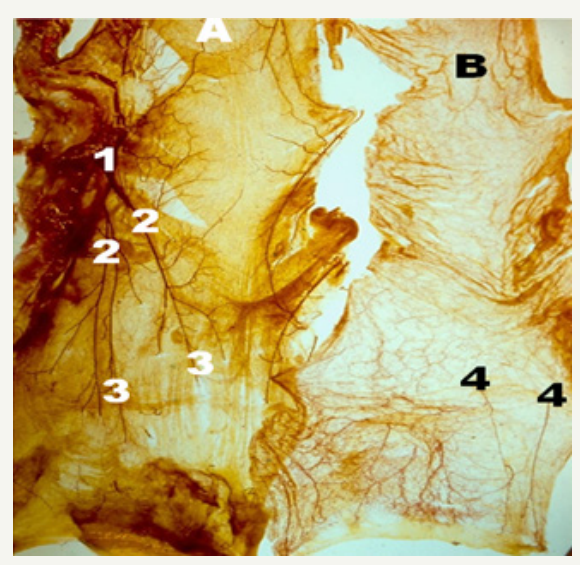

Figure 7: Diaphanization technique of the human rectum previously dissected along the submucosa to separate the muscular layers (A) from the submucosa and mucosa (B): 1-Superior rectal artery; 2-Terminal branches of the superior rectal artery; 3-Here the arteries leave the muscle layer to reach the submucosa; 4-This is where the arteries reach the submucosa.

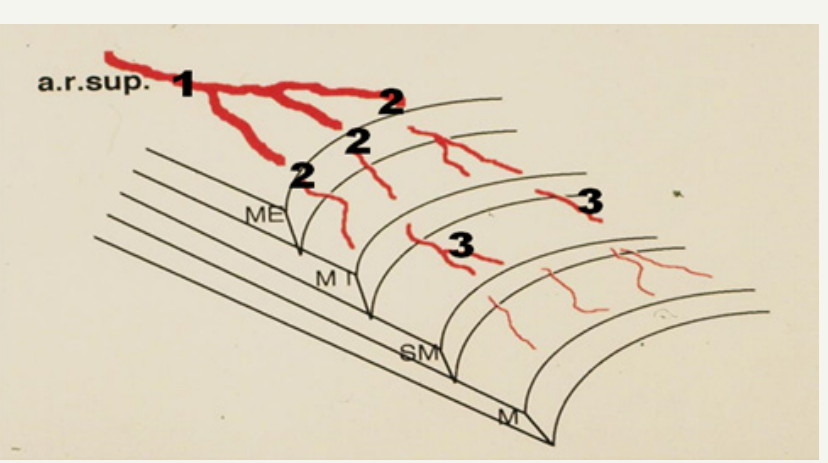

Figure 8: Rectum vascularization scheme: 1-Superior rectal artery; 2-Terminal branches of the superior rectal artery; 3-The arteries reach the submucosa.

ME: Longitudinal musclelayer; MI: Circular muscle layer; SM: Submucosa; M: Mucosa 
The VRB has a lower caliber (almost half) than the VRL Most VRB cross the two muscle layers at the level of mesocolic taeniae. In addition, the small number of VRB that cross only the circular muscle layer have an oblique tract inside the wall of the colon, not like the VRL that describe a straight angle. The intraparietal oblique tract is a protective factor against the formation of diverticula (Figure 6-8)

Rectal diverticula are extremely rare and their cause is still unknown [4]. Three reasons for the low incidence of rectal diverticula have been proposed $[2,4]$. First, the longitudinal muscle fibers of the taenia coli spread outward, thus surrounding the rectum and fortifying its wall against intra-luminal pressure [8]. Second, the internal pressure applied by accumulated feces is less constant in the rectum. Third, the rectum has less peristaltic activity when compared to the sigmoid colon [9]. Focal weak areas in the rectal wall, due to either congenital or acquired factors, can cause rectal diverticula. Possible congenital predisposing factors are: weakness in the circular muscle layer of the rectum, adipose atrophy of the muscle layer of the rectum, as well as deficiencies in the supporting structures such as the coccyx $[4,10]$. Acquired factors are: surgical trauma, excessive relaxation of the recto-vaginal septum, constipation or recurrent impaction causing distension of the rectum, and rectal trauma or infections leading to weakening of the rectal wall $[4,10,11]$. Plavsic et al. [1] reported in 1995 that 2 of 27 patients with scleroderma had rectal diverticulosis without diverticula in the remaining colon [12]. Unlike ARL that weakens the wall of the colon where it crosses perpendicularly and may originate diverticula, the terminal branches of the superior rectal artery cross the two muscle layers of the rectum from the outside to the inside longitudinally and obliquely during a long tract until reaching the submucosa [13]. This long intra parietal oblique tract of the arteries in the thickness of two musclelayers seems sufficient to protect the rectum from diverticular formation.

\section{Conclusion}

The sites where the VRL cross only the circular muscle layer (adjacent to the antimesocolictaenia) are weak points of the colon wall where diverticula can frequently occur. We think that the lower incidence of diverticula in the mesocolic border of the colon is due to the fact that the VRB have a lower caliber than the VRL, more often cross the two muscle layers (in the mesocolictænia level) and, in cases where they cross only the muscle circular layer, they describe a oblique tract during a long path. Diverticula are rare in the rectum not only for the various reasons already described but also because the arteries that vascularize the rectum cross the rectal wall describing a longitudinal and oblique tract during a long path, unlike the VRL of the colon, despite having a mean caliber higher than the VRL.

\section{References}

1. Plavsic BM, Taider L, Drnovsek VH, Kogutt MS (1995) Association of rectal diverticula and scleroderma. Acta Radiol 36(1): 96-9.

2. Maggiori L, Panis Y (2013) Histoirenaturelle de la diverticuloseducôlonetdesmaladiesdiverticulairesducôlon. EMCGastro-entérologie 9(1): 1-7.

3. Sierra EM, Villanueva ES, Fernández JMR, Rocha JLR, Rojas MI (2006) Divertículos rectales. Informe de un caso. Cir Ciruj 74(3): 209-210.

4. Jung SH, Kim JH (2010) A case of solitary rectal diverticulum presenting with a retrorectal mass. Gut Liver 4(3): 394-397.

5. Fagundes RB, Motta GL, Fontana K, Fonseca CB, Binato M (2011) Rectal diverticulum. Arq Bras Cir Dig 24(4): 339-341.

6. Bernardes A, Pina JE, O'Neill JG, Veiga A (1992) Diaphanization: modifications of organ weight and volume. Bull Assoc Anat 76(235): 5355.

7. Bernardes A (1996) Vascularização arterial do cólon sigmoideu e do reto-estudo anatomocirúrgico e experimental. Edição do autor Coimbra $1-314$.

8. Giffin HZ (1911) VII. Diverticulitis of the rectum: a report of two cases operated upon, one of them with carcinomatous degeneration. Ann Surg 53(4): 533-537.

9. Piercy KT, Timaran C, Akin H (2002) Rectal diverticula: report of a case and review of the literature. Dis Colon Rectum 45(8): 1116-1117.

10. Halpert RD, Crnkovich FM, Schreiber MH (1989) Rectal diverticulosis: A case report and review of the literature. Gastrointest Radiol14(1): 274276.

11. Carrim ZI, Murchison JT (2002) Isolated Rectal Diverticula in a Young Man With Idiopathic. Megarectum. Clinical Radiology extra 57: 7-8.

12. Chen CW, Jao SW, Lai HJ, Chiu YC, Kang JC (2005) Isolated rectal diverticulum complicating with rectal prolapse and outlet obstruction: Case report. World J Gastroenterol 11(48): 7697-7699.

13. Patrício J, Bernardes A, Nuno D, Falcão F, Silveira L (1988) Surgical anatomy of the arterial blood-supply of the human rectum. Surg Radiol Anat 10(1): 71-75. 